\title{
Direct Adaptive Control for a Class of Uncertain Nonlinear Systems
}

\author{
Zhenfeng Chen \\ College of Automation \\ Guangdong Polytechnic Normal University \\ No. 293, Zhongshan Avenue, Tianhe District \\ Guangzhou 510665, China
}

\author{
Xuhong Zhang \\ Guangdong Polytechnic \\ Normal University \\ Guangzhou 510665, China
}

\author{
Zhongsheng Wang \\ Guangdong Polytechnic \\ Normal University \\ Guangzhou 510665, China
}

\begin{abstract}
In this paper, a novel systematic design procedure is presented for a class of uncertain nonlinear systems. Such design procedure can remove the control input terms which contain the unknown nonlinearities as the control coefficients, and provides the following advantages: it not only avoids a possible singularity problem completely, but also simplifies the control design process. Moreover, the proposed design procedure can provide simple control structure under the relaxed conditions, which is easy to implement and can be applied to a wider class of systems.
\end{abstract}

\section{General Terms:}

Algorithms, Nonlinear Control Theory

\section{Keywords:}

Adaptive control, Lyapunov function, stability

\section{INTRODUCTION}

Due to its capable of improving a control system performance and stability, adaptive control is a potentially promising technology and has been receiving an increasing amount of attention within control systems society ([1]-[3] and the references therein). Compared with the traditional fixed-gain controller, the distinct feature of adaptive control is the design parameter adaptation which makes it accommodate system uncertainties and improve the control system performance. Nonetheless, such a feature causes a challenge when the control coefficient $b(x)$ is unknown and is approximated by the approximation $\hat{b}(x, \hat{W})$. Although the controlled systems are assumed to be controllable, i.e., $|b(x)| \neq 0$, the approximation model may lose its controllability at some points as $\hat{b}(x, \hat{W}) \rightarrow 0$ during the parameter adaptation period, which is referred to the so-called singularity problem.

For the control of nonlinear system with the unknown control coefficient, additional precautions have to be made to handle the aforementioned problem, such as choosing the initial parameter sufficiently close to the ideal value by off-line training before the operation [4], or applying a projection algorithm to project the estimated parameters in a feasible set, in which $\hat{b}(x, \hat{W}) \neq 0$ (some $a$ priori knowledge is required for the feasible parameter set, and no systematic procedure is available for constructing such a set) [5][8], or requiring the upper bound of the first time derivative of $b(x)$ being known a priori [7], [9]. Recently, several elegant adaptive control schemes were proposed for a special class of nonlinear systems [10 11], where singularity problem was avoided based on the independence of $b(x)$ on $x_{n}$ was assumed. Such structural assumption was removed in [12] by introducing an integral-type Lyapunov function, and a singularity-free adaptive neural controller was provided under the assumptions that $b_{0}$ (i.e., the lower bound of $b(x)$ ) and some extra $a$ priori knowledge were needed. Due to the integral operation, this method led to complex and difficult to implement controllers. Improvements on the result of [12] were addressed in [13, 14], and drawn two points. The one point was proposed adaptive controllers with lower dimensionality of neural networks, the other point was relaxed certain restrictions in [12], such as $b_{0}$ was not required to be known in [13 14], and some knowledge in [12] were relaxed to be unknown in [13].

This paper follows up the works of [10]-[14], and presents a novel systematic procedure for the design of a new singularity-free adaptive control. All the signals in the closed-loop system are guaranteed to be bounded and the output of the systems is proven to converge to a small neighborhood of the desired trajectory. The relationship between the transient performance and the design parameters is explicitly given to guide the tuning of the controller.

The main contributions of this paper are as follows.

(i) For the considered systems, the control input term $b(x) u$ is divided into two parts- $b_{0} u$ and $\left(b(x)-b_{0}\right) u$. A systematic procedure is developed for the design of an adaptive control such that, for the derivatives of Lyapunov function candidates, the former part can be guaranteed to be stabilized and the latter part can be guaranteed to be non-positive. Due to the negative semi-definiteness, the latter part can be removed in the derivatives of Lyapunov function candidates, which not only simplifies the control design process, but also tackles the aforementioned possible singularity problem without using projection algorithms [5], 6], or the upper bound of the first time derivative of $b(x)$ [7], [9].

(ii) Compared with the results in [12]-[14], the stabilities and control performances in this paper are achieved without the assumptions that $b_{0}$ is required to be known in [12], or some knowledge on unknown function is known a priori in [12]-[14]. 
(iii) The proposed design procedure results in more simple control structures than that of [10]-[14], which implies that the proposed controllers are easier to implement and more reliable for practical purposes.

The rest of the paper is organized as follows. Section 2 presents the problem formulation. In Section 3 we describe the proposed adaptive control along with the main theoretical results. Section 4 provides a simulation example to illustrate the effectiveness of the proposed approach. Finally, in Section 5 we draw our conclusion.

\section{PROBLEM STATEMENT}

Consider the adaptive control problem for nonlinear systems transformable to the following canonical form

$$
\left\{\begin{array}{l}
\dot{x}_{i}=x_{i+1}, \quad i=1,2, \cdots, n-1 \\
\dot{x}_{n}=a(x)+b(x) u \\
y=x_{1}
\end{array}\right.
$$

where $x=\left[x_{1}, x_{2}, \cdots, x_{n}\right]^{T} \in \mathbb{R}^{n}, u \in \mathbb{R}$, and $y \in \mathbb{R}$ are the state variables, system input and output, respectively; $a(x)$ and $b(x)$ are unknown smooth functions. The control objective is to synthesize an adaptive tracking control for system (1) such that the output $y$ tracks a desired smooth trajectory $y_{d}$.

ASSUMPTION 1. The sign of $b(x)$ is known, and there exists an unknown constant $b_{0}>0$ such that $|b(x)| \geq b_{0}, \forall x \in \mathbb{R}^{n}$.

In the above assumption, $|b(x)| \geq b_{0}$ poses a controllable condition on system (1), and implies that the smooth function $b(x)$ is strictly either positive or negative. Without loss of generality, assume that $b(x) \geq b_{0}>0, \forall x \in \mathbb{R}^{n}$.

REMARK 1. Assumption 1 is more relaxed than the assumptions made in many control schemes (Kristic et al. [15], Sepulchre et al. [16], Ge et al. [17] and references therein), where, besides the conditions in Assumption 1, a $a(x)$ is required to be bounded by a known continuous function [12] 14]-[17], and in [12] $b_{0}$ is even required to be known a priori.

ASSUMPTION 2. $x_{d}=\left[y_{d}, \dot{y}_{d}, \cdots, y_{d}^{(n-1)}\right]^{T}$ is available, and $x_{d} \in \Omega_{x_{d}}, \forall t \geq 0$, with $\Omega_{x_{d}} \subset \mathbb{R}^{n}$ being a compact set.

\section{ADAPTIVE CONTROL DESIGN AND STABILITY ANALYSIS}

In this section, two singularity-free direct adaptive control schemes are presented without using integral-type Lyapunov functions. A new control scheme is first proposed to develop a simple control algorithm. Then another control scheme is proposed to result in a lower dimensions of function approximators.

For the control of system (1), define the tracking error $e_{1}=y-y_{d}$ and

$$
\begin{aligned}
& e=x-x_{d}=\left[e_{1}, e_{2}, \cdots, e_{n}\right]^{T} \\
& s=\left(\frac{d}{d t}+\lambda\right)^{n-1} e_{1}=\left[\begin{array}{ll}
\Lambda^{T} & 1
\end{array}\right] e
\end{aligned}
$$

where $\Lambda=\left[\lambda^{n-1},(n-1) \lambda^{n-2}, \cdots,(n-1) \lambda\right]^{T}$ with constant $\lambda>0$.

From (1) and [2], the time derivative of $s$ can be written as

$$
\dot{s}=a(x)+b(x) u+v
$$

where $v=-y_{d}^{(n)}+\left[\begin{array}{ll}0 & \Lambda^{T}\end{array}\right] e$.
REMARK 2. As mentioned in [12] 20], the tracking error $e_{1}$ in (2) can be expressed as $e_{1}=H(s) s$, with $H(s)$ a proper stable transfer function, which has the following properties: (i) on the time-varying hyperplan $s=0$ in $\mathbb{R}^{n}$, $e_{1}$ converges to zero asymptotically; (ii) if $e(0) \in \Omega_{e}$ and $|s(t)| \leq c, \forall t \geq 0$, with constant $c>0$ and

$$
\Omega_{e}=\left\{e:\left|e_{i}\right| \leq 2^{i-1} \lambda^{i-n} c, i=1,2, \cdots, n\right\}
$$

then, $e(t) \in \Omega_{e}, \forall t \geq 0$; (iii) if $e(0) \notin \Omega_{e}$ and $|s(t)| \leq c, \forall t \geq 0$, then $e(t)$ will converge to $\Omega_{e}$ within a time-constant $(n-1) / \lambda$ and remain inside $\Omega_{e}$.

Constructing a Lyapunov function candidate $V_{s}=(1 / 2) s^{2}$, its derivative is

$$
\dot{V}_{s}=b_{0} s\left[\bar{a}(x, v)+u+\bar{b}^{+}(x) u\right]
$$

where $\bar{a}(x, v)=\frac{a(x)+v}{b_{0}}, \bar{b}^{+}(x)=\frac{b(x)}{b_{0}}-1>0$.

The basic idea of the control design in this paper is to guarantee $V_{s}$ to be a Lyapunov function by setting the terms involved in (4) suitably. This can be accomplished by choosing $u^{*}$ such that (i) $u^{*}=-k s-\bar{a}(x, v)$, where $k>0$ is a design constant, and (ii) $\bar{b}^{+}(x) s u^{*} \leq 0$. After these manipulations, $V_{s}$ becomes a Lyapunov function, and $s=0$ is thus asymptotically stable.

\subsection{Control Scheme I}

Since $a(x)$ and $b_{0}$ are unknown, $\bar{a}(\cdot)$ in $u^{*}$ is an unknown smooth function of $x$ and $v$. Due to their great capabilities in function approximation, several function approximators can be applied for approximating the unknown smooth function, e.g., radial basis function neural networks, high-order neural networks or fuzzy systems. Such approximators can be described as $W^{T} S(Z)$, where $Z \in \Omega_{Z} \subset \mathbb{R}^{q}$ is the input vector, $W \in \mathbb{R}^{l}$ is the weight vector, $l>1$ is the node number, and $S(Z) \in \mathbb{R}^{l}$ is the basis function vector. Universal approximation results indicate that any continuous function over a compact set $Z \in \Omega_{Z} \subset \mathbb{R}^{q}$ can be approximated to any arbitrary accuracy by using $W^{T} S(Z)$ and choosing $l$ sufficiently large. Thus, $\bar{a}(x, v)$ can be written as

$$
\bar{a}(x, v)=W^{* T} S(Z)+\varepsilon, \forall Z=\left[\begin{array}{ll}
x & v
\end{array}\right]^{T} \in \Omega_{Z}
$$

where $\varepsilon$ is the approximation error, $W^{*}$ is the ideal constant weights such that $|\varepsilon| \leq \varepsilon^{*}, \forall Z \in \Omega_{Z}$ with constant $\varepsilon^{*}>0$.

REMARK 3. Since signals $x$ and $x_{d}$ are known, $v=-y_{d}^{(n)}+$ $\left[\begin{array}{ll}0 & \Lambda^{T}\end{array}\right]$ e is available. To use less neurons, $[x v] \in \mathbb{R}^{n+1}$ is chosen as the input to $W^{T} S(Z)$ rather than $\left[x x_{d}\right] \in \mathbb{R}^{2 n}$. Thus, the online computation load is lightened.

Design the control input $u$ as

$$
u=-k s-\varpi \hat{W}^{T} S(Z)
$$

where $\hat{W}$ is the estimate of neural weights $W^{*}$, and $\varpi=\tanh \left(\frac{\omega}{\epsilon}\right)$ with $\omega=\hat{W}^{T} S(Z) s$ and a small constant $\epsilon>0$.

According to Assumption 1 the following inequality holds

$$
\bar{b}^{+}(x) s u=-\bar{b}^{+}(x)\left[k s^{2}+\tanh \left(\frac{\omega}{\epsilon}\right) \omega\right] \leq 0
$$

Consider a Lyapunov function candidate $V$ as

$$
V=\frac{1}{2} s^{2}+\frac{b_{0}}{2} \tilde{W}^{T} \Gamma^{-1} \tilde{W}
$$


where $\tilde{W}=\hat{W}-W^{*}$, and $\Gamma=\Gamma^{T}>0$ is an adaptation gain matrix.

Using (4)-(7), the derivative of $V$ is

$$
\begin{array}{r}
\dot{V} \leq b_{0}\left[-k s^{2}+W^{* T} S(Z) s-\varpi \omega\right. \\
\left.+\tilde{W}^{T} \Gamma^{-1} \dot{\hat{W}}+s \varepsilon\right]
\end{array}
$$

Consider the facts that

$$
\begin{aligned}
& W^{* T} S(Z) s-\varpi \omega+\tilde{W}^{T} \Gamma^{-1} \dot{\hat{W}} \\
= & \omega-\tanh \left(\frac{\omega}{\epsilon}\right) \omega+\tilde{W}^{T} \Gamma^{-1}[\dot{\hat{W}}-\Gamma S(Z) s]
\end{aligned}
$$

and the following nice property of function $\tanh (\cdot)[21]$ :

$$
0 \leq|\omega|-\omega \tanh \left(\frac{\omega}{\epsilon}\right) \leq 0.2785 \epsilon, \forall \epsilon>0, \forall \omega \in \mathbb{R}
$$

Design adaptation law for $\hat{W}$ as

$$
\dot{\hat{W}}=\Gamma[S(Z) s-\sigma|s| \hat{W}]
$$

where $\sigma>0$ is a design parameter.

LEMMA 1. For adaptive algorithm [12, there exists a compact set

$$
\Omega_{\hat{W}}=\left\{\hat{W}:\|\hat{W}\| \leq \frac{c_{N N}}{\sigma}\right\}
$$

such that if $\hat{W}(0) \in \Omega_{\hat{W}}$, then $\hat{W}(t) \in \Omega_{\hat{W}}, \forall t \geq 0$, where $\|S(Z)\| \leq c_{N N}$ with constant $c_{N N}>0$.

PROOF. similar to the proof procedure in $[14, \mathrm{Ch} .8]$.

From (11) and (12), (10) becomes

$$
\begin{aligned}
W^{* T} S(Z) s & -\varpi \omega+\tilde{W}^{T} \Gamma^{-1} \dot{\hat{W}} \\
& \leq 0.2785 \epsilon-\sigma|s| \tilde{W}^{T} \hat{W}
\end{aligned}
$$

Using Young's inequality [18], we have

$$
\begin{aligned}
-\sigma|s| \tilde{W}^{T} \hat{W} & \leq-\sigma|s|\|\tilde{W}\|^{2}+\sigma|s|\|\tilde{W}\|\left\|W^{*}\right\| \\
& \leq \frac{\sigma|s|\left\|W^{*}\right\|^{2}}{4} \leq \frac{k s^{2}}{4}+\frac{\sigma^{2}\left\|W^{*}\right\|^{4}}{16 k} \\
s \varepsilon & \leq \frac{k}{4} s^{2}+\frac{1}{k} \varepsilon^{2}
\end{aligned}
$$

Substituting (14)-(16) into (9), we have

$$
\dot{V} \leq-\frac{b_{0} k}{2} s^{2}+\frac{b_{0}}{k} \varepsilon^{2}+\eta
$$

where $\eta=b_{0}\left(0.2785 \epsilon+\frac{\sigma^{2}\left\|W^{*}\right\|^{4}}{16 k}\right)$.

Based on the above analysis, the following theorem states the stability and control performance of the closed-loop system.

THEOREM 2. Consider the closed-loop adaptive system consisting of the plant (17) satisfying Assumptions 1 and 2, the controller (6) and the weight updating law (12). Assume that there exists sufficiently large compact set $\Omega_{Z}$ such that $Z \in \Omega_{Z}, \forall t \geq 0$. Then, for bounded initial conditions, (i) all the signals in the closed-loop system are bounded, and there exists a constant $T>0$, for all $t \geq T$ the state vector $x$ remain in

$$
\Omega_{\varrho}=\left\{x:\left|e_{i}\right| \leq 2^{i-1} \lambda^{i-n} \varrho, i=1,2, \cdots, n, x_{d} \in \Omega_{x_{d}}\right\}
$$

where $\varrho=\sqrt{\frac{2}{k}\left(0.2785 \epsilon+\frac{\sigma^{2}\left\|W^{*}\right\|^{4}}{16 k}+\frac{\varepsilon^{* 2}}{k}\right)}$.

(ii) the mean square of output tracking error satisfies

$$
\lim _{t \rightarrow \infty} \frac{1}{t} \int_{0}^{t} s^{2} d \tau \leq \rho
$$

where $\rho=\frac{2 \eta}{b_{0} k}+\frac{2}{k^{2}} \lim _{t \rightarrow \infty} \frac{1}{t} \int_{0}^{t} \varepsilon^{2} d \tau$.

Proof. (i) From 17, $\dot{V}<0$ when $s$ outside the compact set:

$$
\Omega_{s}=\{s:|s| \leq \varrho\}
$$

where $\varrho$ is defined in 18 .

Considering Lemma 1 and following the boundedness theorem (e.g., Theorem 2.14 in [19]), we obtain that $s$ and $\hat{W}$ are uniformly ultimately bounded. From Remark 2.1 in [12], the boundedness of $s$ implies that there exists a computable constant $T>0$, for all $t \geq T$ the state vector $x$ remains in $\Omega_{\varrho}$ defined in (18). Using (6), control $u$ are also bounded. Thus, all the signals in the closed-loop system remain bounded.

(ii) Integrating 17 over $[0, t]$ leads to

$$
\frac{b_{0} k}{2} \int_{0}^{t} s^{2} d \tau \leq V(0)-V(t)+\frac{b_{0}}{k} \int_{0}^{t} \varepsilon^{2} d \tau+\eta t
$$

Noting that the positivity of $V, 21$ follows that

$$
\int_{0}^{t} s^{2} d \tau \leq \frac{2}{b_{0} k} V(0)+\frac{2}{k^{2}} \int_{0}^{t} \varepsilon^{2} d \tau+\frac{2}{b_{0} k} \eta t
$$

which proves 19

\subsection{Control Scheme II}

To further reduce the dimensionality of the input of the approximator (5), we rewrite (3) as

$$
\dot{s}=b_{0}\left[\frac{a(x)}{b_{0}}+\frac{v}{b_{0}}+u+\bar{b}^{+}(x) u\right]
$$

where $\bar{b}^{+}(x)$ is defined in (4).

Lemma 3. For (23) satisfying Assumptions 1 and 2 with assuming that $a(x)$ and $b_{0}$ are known exactly and $\bar{b}^{+}(x)$ su $\leq 0, \forall t \geq$ 0 , if a desired controller is designed as

$$
\bar{u}^{*}=-\bar{k} s+\bar{u}_{1}^{*}
$$

where $\bar{u}_{1}^{*}=-\frac{a(x)}{b_{0}}, \bar{k}=k+k_{v} v^{2}>0$ with $k_{v}>0$ being $a$ design constant and $k>0$ defined previously, then s converges to an adjustable neighborhood of zero.

PROof. Consider $V_{s}=(1 / 2) s^{2}$ and the assumption that $\bar{b}^{+}(x) s u \leq 0, \forall t \geq 0$. Its time derivative

$$
\begin{aligned}
\dot{V}_{s} & =b_{0} s\left[-\bar{k} s+\frac{v}{b_{0}}+\bar{b}^{+}(x) u\right] \\
& \leq b_{0}\left[-k s^{2}-k_{v} v^{2} s^{2}+\frac{|v s|}{b_{0}}\right]
\end{aligned}
$$


Using Young's inequality [18], we have

$$
\frac{|v s|}{b_{0}} \leq k_{v}(v s)^{2}+\frac{1}{4 k_{v} b_{0}^{2}}
$$

Substituting (25) into 26) yields

$$
\dot{V}_{s} \leq-2 k b_{0} V_{s}+\frac{1}{4 k_{v} b_{0}}
$$

This implies that $s$ eventually converges to the compact set

$$
\Omega_{s}=\left\{s: V_{s} \leq \frac{1}{8 k k_{v} b_{0}^{2}}\right\}
$$

where $k_{v}, k$ are design parameters.

Since $a(x)$ and $b_{0}$ are unknown, $\bar{u}_{1}^{*}$ in 24 is an unknown smooth function of $x$. Thus, $\bar{u}_{1}^{*}$ can be approximated by employing $W^{T} S(x)$, i.e., $\bar{u}_{1}^{*}$ can be written as

$$
\bar{u}_{1}^{*}=W^{* T} S(x)+\varepsilon, x \in \Omega_{x}
$$

where $W^{*}$ and $\varepsilon$ is defined in (5).

Motivated by the desired controller structure 24, the control input $u$ (6) can be modified as

$$
u=-\bar{k} s-\varpi \hat{W}^{T} S(x)
$$

According to Assumption 1 the following inequality holds

$$
\bar{b}^{+}(x) s u=-\bar{b}^{+}(x)\left[\bar{k} s^{2}+\tanh \left(\frac{\omega}{\epsilon}\right) \omega\right] \leq 0
$$

Under the control 30 and proceeding in the same manner as in 3.1 it is not difficult to prove the following theorem.

THEOREM 4. Consider the closed-loop adaptive system consisting of the plant (1) satisfying Assumptions 1 and 2 , the controller (30) and the weight updating law (12). Then, for bounded initial conditions,

(i) all the signals in the closed-loop system are bounded, and and there exists a constant $T>0$, for all $t \geq T$ the state vector $x$ remain in

$$
\Omega_{\bar{\varrho}}=\left\{x:\left|e_{i}\right| \leq 2^{i-1} \lambda^{i-n} \bar{\varrho}, i=1,2, \cdots, n, x_{d} \in \Omega_{x_{d}}\right\}
$$

where $\varrho=\sqrt{\varrho^{2}+\frac{1}{k k_{v} b_{0}^{4}}-\frac{2 k_{v} v^{2}}{k^{2}\left(k+k_{v} v^{2}\right)}\left(\varepsilon^{* 2}+\frac{\sigma^{2}\left\|W^{*}\right\|^{4}}{16}\right)}$ with $\varrho$ being defined in 18 .

(ii) the mean square of output tracking error satisfies

$$
\lim _{t \rightarrow \infty} \frac{1}{t} \int_{0}^{t} s^{2} d \tau \leq \bar{\rho}
$$

where $\bar{\rho}=\rho+\frac{1}{k k_{v} b_{0}^{4}}-\frac{2 k_{v} v^{2}}{k^{2}\left(k+k_{v} v^{2}\right)}\left(\lim _{t \rightarrow \infty} \frac{1}{t} \int_{0}^{t} \varepsilon^{2} d \tau+\right.$ $\left.\frac{\sigma^{2}\left\|W^{*}\right\|^{4}}{16}\right)$.

\section{SIMULATION STUDIES}

To illustrate the effectiveness of the proposed control approach, the following nonlinear system is considered

$$
\left\{\begin{array}{l}
\dot{x}_{1}=x_{2} \\
\dot{x}_{2}=a(x)+b(x) u+d(t) \\
y=x_{1}
\end{array}\right.
$$

where $a(x)=-4\left(\sin \left(4 \pi x_{1}\right) /\left(\pi x_{1}\right)\right)\left(\sin \left(\pi x_{2}\right) /\left(\pi x_{2}\right)\right)^{2}, b(x)=$ $2-\sin \left(3 \pi\left(x_{1}-0.5\right)\right)$ and $d(t)=0.1 \cos (0.01 t) \cos \left(x_{1}\right)$.
The control objective is to make the outputs $y$ tracks the desired reference trajectories $y_{d}$, which are the outputs of the famous van der Pol oscillator [22]

$$
\left\{\begin{array}{l}
\dot{x}_{d 1}=x_{d 21} \\
\dot{x}_{d 2}=-x_{d 1}+\beta\left(1-x_{d 1}^{2}\right) x_{d 2} \\
y_{d}=x_{d 1}
\end{array}\right.
$$

where the output $y_{d}$ approaches a limit cycle when $\beta>0$.

The adaptive controllers and the design parameters for system 34 are chosen as follows:

$$
u=-k s-\varpi \hat{W}^{T} S(Z)
$$

where $s=-y_{d}^{2}-[0 \lambda]^{T}\left(x-x_{d}\right), \varpi=\tanh \left(\frac{\hat{W}^{T} S(Z) s}{\epsilon}\right)$ and $\hat{W}$ are updated by $\dot{\hat{W}}=\Gamma[S(Z) s-\sigma|s| \hat{W}]$ with $Z=[x, v]^{T} \in \mathbb{R}^{3}$, $k=2, \Gamma=\operatorname{diag}\{2.0\}, \sigma=0.1, \epsilon=0.1$. In the following simulation studies, $\hat{W}^{T} S(Z)$ is constructed using neural network, which contains 27 nodes (i.e., $l=27)$, with widths $v_{k}=2(k=$ $1,2, \cdots, l)$ and centers $\mu_{k}(k=1,2, \cdots, l)$ evenly spaced in $[-2.5,2.5] \times[-2,2]$.

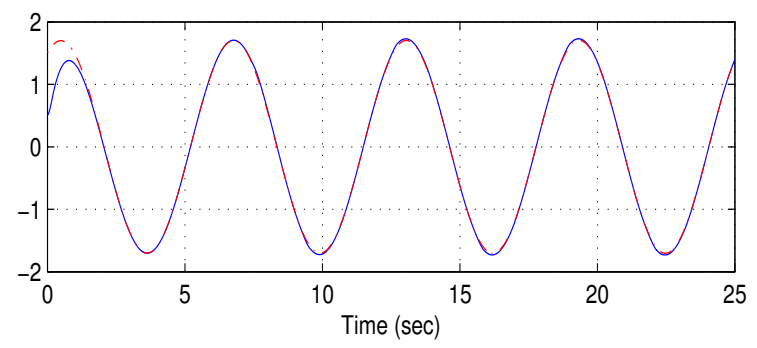

Fig. 1. Output $y_{1}$ (“-”) follows $y_{r 1}($ (“- -")

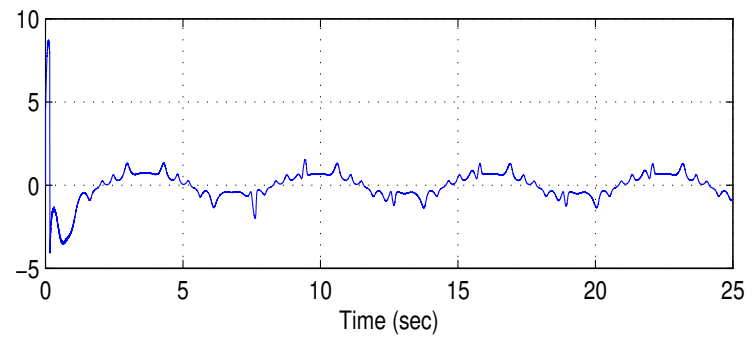

Fig. 2. Control input $u$

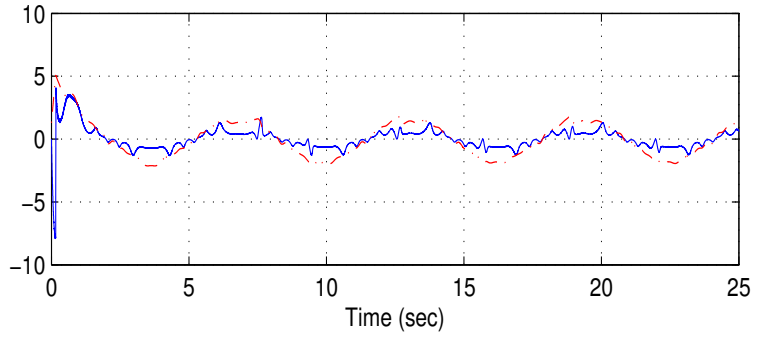

Fig. 3. Unknown function $\nu$ (“-”) and its estimate $\varpi W^{T} S(Z)$ (“- -”)

Figs. 1-3 show the simulation results of applying controller $(36)$ to system (34) for tracking reference signals $y_{d}$ with $\beta=0.001$ and the initial conditions $x=[0.5 ; 2 ; 1.3], x_{d}=[1.5 ; 0.8], \hat{W}=0$. Figs. 1 show the fairly good tracking performance. From Figs. 2, it follows that the control signals $u$ is bounded and become periodic signals after 2s. Figs. 3 illustrate the learning ability of neu- 
ral networks by plotting the nonlinear function as well as its estimate. Note that the tracking performance improves with increase of matching between the nonlinear function and its estimate. Hence, the proposed adaptive controller possesses the abilities of learning and controlling the unknown nonlinear system.

\section{CONCLUSION}

For a class of uncertain nonlinear systems, this paper have presented a novel systematic design procedure, which not only eliminates the possible singularity problem completely, but also simplifies the control design process and provides simple control structure. The proposed controllers are easy to implement and can be applied to a wider class of systems due to their relaxed conditions. In the future, investigation on a general class of nonaffine nonlinear systems will be interesting research topics in this field.

\section{ACKNOWLEDGEMENTS}

Supported by National Natural Science Foundation of China (61374003), Foundation for Distinguished Young Talents in Higher Education of Guangdong Province of China (2014KQNCX173), Doctor scientific research of Guangdong Polytechnic Normal University, Project of Guangdong Polytechnic Normal University (14KJY12), Foundation for 1st batch Distinguished Talents in Higher Education of Guangdong Province of China, 2013'.

\section{REFERENCES}

[1] Chen Z. F., Ge S. S., Zhang Y., Li Y. 2014. Adaptive neural control of MIMO nonlinear systems with a block-triangular pure-feedback control structure. IEEE Transactions on Neural Networks and Learning Systems. 25(11), 2017-2029.

[2] Chen Z. F., Zhang Y. 2014. Robust control of a class of nonaffine nonlinear systems by state and output feedback. Journal of Central South University, 21(4), 1322-1328.

[3] Zhang L., Li K., Bai E. W. 2013. A new extension of newton algorithm for nonlinear system modelling using RBF neural networks. IEEE Trans. Automat. Contr. 58(11), 2929-2933.

[4] Chen F. C., Liu C. C. 1994. Adaptively controlling nonlinear continuous-time systems using multilayer neural networks. IEEE Trans. Automat. Contr. 39, 1306-1310.

[5] Sastry S. S., Isidori A. 1989. Adaptive control of linearizable systems. IEEE Trans. Automat. Contr. 34, 1123-1131.

[6] Polycarpou M. M., Ioannou P. A. 1992. Modeling, identification and stable adaptive control of continuous-time nonlinear dynamical system using neural networks. Proc. Am. Contr. Conf. Chicago, IL, 36-40.

[7] Wang L. X. 1994. Adaptive Fuzzy Systems and Control: Design and Analysis. Englewood Cliffs, NJ: Prentice-Hall.

[8] Spooner J. T., Passino K. M. 1996. Stable adaptive control using fuzzy systems and neural networks. IEEE Trans. Fuzzy Syst. 4, 339-359.

[9] Sanner R. M., Slotine J. E. 1992. Gaussian networks for direct adaptive control. IEEE Trans. Neural Networks, 3, 837-863.

[10] Ge S. S., Hang C. C., Zhang T. 1999. A direct method for robust adaptive nonlinear control with guaranteed transient performance. Systems \& Control Letters. 37, 275-284.

[11] Zhang T., Ge S. S., Hang C. C. 1999. Design and performance analysis of a direct adaptive controller for nonlinear systes. Automatica. 35, 1809-1817.
[12] Zhang T., Ge S. S., Hang C. C. 2000. Stable adaptive control for a class of nonlinear systems using a modified Lyapunov function. IEEE Trans. Automat. Contr. 45, 129-132.

[13] Huang S. N., Tan K. K., Lee T. H. 2003. Further results on adaptive control for nonlinear systems using neural networks. IEEE Trans. Neural Netw. 14(3), 129-132.

[14] Huang S. N., Tan K. K., Lee T. H. 2004. An improvement on stable adaptive control for a class of nonlinear systems. IEEE Trans. Automat. Contr. 49(8), 1398-1403.

[15] Krstić M., Kanellakopoulos I., Kokotović P. V. 1995. Nonlienar and Adaptive Control Design. New York: Wiley.

[16] Sepullchre R., Jankovic M., Kokotovic P. V. 1997. Constructive nonlinear control. London, U.K.:Springer-Verlag.

[17] Ge S. S., Hang C. C., Lee T. H., Zhang T. 2001. Stable Adaptive Neural Network Control. Norwell, MA: Kluwer.

[18] Young W. H. 1912. On the multiplication of successions of Fourier constants. Proc. Roy. Soc. Lond. Series A, 87(596), 331-339.

[19] Qu Z. 1998. Robust Control of Nonlinear Uncertain Systems. New York: Wiley.

[20] Slotine J. E., Li W. 1991. Applied Nonlinear Control. Englewood Cliffs, NJ: Prentice-Hall.

[21] Polycarpou M. M., Ioannou P. A. 1995. A robust adaptive nonlinear control design. Automatica. 32(3), 423-427.

[22] Vidyasagar M. 1993. Nonlinear Systems Analysis, 2nd ed. Englewood Cliffs, NJ: Prentice-Hall. 